\title{
Effect of platelet-activating factor (PAF) on human spermatozoa-oocyte interactions
}

\author{
M. J. Angle ${ }^{1, *}$, R. Tom ${ }^{2}$, K. Jarvi ${ }^{2, * *}$ and R. D. McClure ${ }^{2, * * *}$ \\ Department of ${ }^{1}$ Obstetrics and Gynecology and ${ }^{2}$ Urology, University of California Medical School, \\ San Francisco, CA 94114, USA
}

\begin{abstract}
The effect of exogenous platelet-activating factor (PAF) on human spermatozoa was examined by monitoring acrosomal status and hamster oocyte penetration. Induction of the acrosome response by PAF was dose, and time, dependent and required capacitated spermatozoa. Addition of $10^{-14} \mathrm{~mol} \mathrm{PAF} \mathrm{l}^{-1}$ enhanced the acrosome reaction eightfold compared with controls treated with human serum albumin (HSA). Similar concentrations of lyso-PAF failed to induce acrosomal loss and preincubation of spermatozoa with CV3988, an inhibitor of PAF, prevented PAF induction of the acrosome reaction. PAF significantly increased the number of zona-free hamster oocytes penetrated compared with controls $(9.8 \pm 0.5$ of 25 oocytes were penetrated by control spermatozoa compared with $23.3 \pm 0.8$ out of 25 oocytes penetrated after incubation of spermatozoa with $10^{-14} \mathrm{~mol} \mathrm{PAFl}^{-1} ; 93 \%$ of all oocytes were penetrated by at least one spermatozoon following incubation with PAF), and also increased the number of decondensed spermatozoa found per egg during the sperm penetration assay (from $1.7 \pm 0.3$ spermatozoa/egg with control spermatozoa to $3.3 \pm 0.5$ spermatozoa/egg with PAF-treated spermatozoa). PAF-induced increases in acrosome reaction and sperm penetration assay values were similar to effects obtained with human follicular fluid and were calcium dependent. Induction of the acrosome reaction by physiological concentrations of PAF appeared to be morphologically similar to the response induced by follicular fluid as assessed by electron microscopy.
\end{abstract}

\section{Introduction}

Members of a family of biologically active phospholipids, collectively known as platelet-activating factor (PAF; 1-O-alkyl2-acetyl-sn-glyceryl-3-phosphocholine), have been shown to modulate a number of physiological effects, including systemic vasodilation, pulmonary bronchiole constriction, platelet and neutrophil activation, initiation of cardiac ischaemia, tissue rejection and gastric ulcer formation (Braquet et al., 1987).

Recently PAF has been linked with various reproductive processes. Several studies implicate PAF in events associated with follicular development and ovulation in the female: intraovarian instillation of PAF has been shown to induce ovulation, and the use of PAF antagonists blocks follicular rupture (Abisogun et al., 1989); PAF has been found in human follicular fluid; and addition of exogenous PAF to human granulosa cell cultures modulates progesterone biosynthesis (Rabinovici and Angle, 1991). PAF also appears to mediate events directly associated with fertilization: addition of PAF increases the fertilization rate of mouse oocytes in vitro, and the use of PAF antagonists can block fertilization in vivo as well as in vitro (Harper et al., 1989; Minhas et al., 1989; Kuzan, 1990). It has been proposed that

${ }^{*}$ Present address and reprint requests: Virginia Mason Medical Center, 1100 9th Ave., PO Box 900, X11-FC, Seattle, WA 98111, USA; ${ }^{* *}$ Present address: Royal Victoria Hospital, Montreal PQ, Canada; ${ }^{* *}$ Present address: Virginia Mason Medical Center, 1100 9th Ave., PO Box 900, C7-S, Seattle, WA 98111, USA. Received 18 September 1992.
PAF is the first embryonic signal of pregnancy (O'Neill, 1985a, b, c; Orozco et al., 1986), and embryonic production of PAF may reflect embryo health and viability (O'Neil and Saunders, 1985; O’Neill et al., 1985, 1987; O'Neill, 1987; Punjabi et al., 1990). PAF also appears to be associated with implantation, as PAF concentrations in the uterus change during the implantation period (Angle et al., 1988a); administration of PAF antagonists inhibits implantation (Acker et al., 1988; Spinks and $\mathrm{O}^{\prime}$ Neill, 1988), and injection of PAF into the uterus induces a decidua-like reaction in pseudopregnant rats (Acker et al., 1989). PAF may also play a role in the maintenance of pregnancy (Geissler et al., 1989). Finally, during the final stages of pregnancy, increasing concentrations of PAF are found in amniotic fluid and appear to be associated with the onset of parturition (for review, see Johnston et al., 1990).

PAF also plays a role in male reproductive physiology and has been reported in spermatozoa from normal men, rabbits and mice (Kumar et al., 1988; Minhas et al., 1989; Kuzan et al., 1990), although Collier et al. (1990) failed to detect PAF in human spermatozoa using a platelet-aggregation assay. It has also been reported that PAF increases the motility of spermatozoa from normal human donors (Ricker et al., 1989). However, other investigators have been unable to observe an effect of PAF on the motility of spermatozoa from normal donors or subfertile patients either before or after overnight capacitation (Angle et al., 1991). However, since PAF antagonists appear to be spermicidal (Harper et al., 1989) and can inhibit fertilization, PAF 
may affect other aspects of sperm function without modulating sperm motility. These experiments were therefore designed to examine the role of PAF in spermatozoa-egg interactions by monitoring the effect of exogenous PAF on initiation of the acrosome reaction and on hamster oocyte penetration.

\section{Materials and Methods}

\section{Semen collection and preparation}

Semen was collected from normal donors routinely used as controls for clinical tests in the Andrology Laboratory at the University of California, San Francisco. Permission to use the donated spermatozoa for experimental purposes was obtained from each patient and from the Research Evaluation and Allocation Committee. Semen was washed twice with Biggers, Whitten and Whittingham (BWW) media supplemented with $0.6 \%$ human serum albumin (HSA; Fraction V, Sigma, St Louis, $\mathrm{MO}$ ), pelleted, and overlayered with BWW to obtain a 'swim-up' population of motile spermatozoa. This population of spermatozoa was either used immediately (noncapacitated spermatozoa) or incubated overnight at $37^{\circ} \mathrm{C}$; the spermatozoa were then used to assess the effect of PAF on the acrosome reaction or on spermatozoa-egg interaction in the zona-free hamster oocyte penetration assay.

\section{Preparation of PAF}

PAF was prepared by resuspending the original sample, from Avanti Polar Lipids (Birmingham, $\mathrm{AL}$ ), in chloroform:methanol 2:1 and storing it at $-20^{\circ} \mathrm{C}$ until phosphorus analysis was completed (Rouser et al., 1966). After quantitation of the phosphorus content, an aliquot of the chloroform:methanol solution was removed, dried under a stream of nitrogen at $37^{\circ} \mathrm{C}$, then resuspended in $0.25 \% \mathrm{HSA}$ in $0.9 \%$ saline to give the required concentration of PAF. Lyso-PAF was treated in a similar manner.

\section{Assessment of acrosome reaction}

The effect of PAF or lyso-PAF on the spermatozoa acrosome was evaluated by staining with fluorescein isothiocyanate (FITC)-labelled Pisum sativum agglutinin (PSA) (Vector Labs, Inc., Burlingame, CA) as described by Cross et al. (1986). Briefly, washed, swim-up spermatozoa that had been capacitated overnight in BWW containing $0.6 \%$ HSA were incubated with various concentrations of PAF $\left(10^{-9}-10^{-16} \mathrm{~mol} \mathrm{I}^{-1}\right)$, lyso-PAF $\left(10^{-14} \mathrm{~mol}^{-1}\right)$ or $0.25 \%$ HSA in physiological saline (PAF control) for $30 \mathrm{~min}$. When the PAF inhibitor CV3988 was used, spermatozoa were incubated with the inhibitor $\left(10^{-12} \mathrm{~mol} \mathrm{l}^{-1}\right)$ for $30 \mathrm{~min}$ before addition of PAF $\left(10^{-14} \mathrm{~mol} \mathrm{l}^{-1}\right)$. Extraneous protein was removed by washing, and the spermatozoa fixed in $95 \%$ ethanol for $45 \mathrm{~min}$ at $4^{\circ} \mathrm{C}$. A smear of spermatozoa was then made and combined with $30 \mu \mathrm{g}$ FITC-labelled PSA ml${ }^{-1}$ for $2 \mathrm{~min}$. Coverslips were mounted using $1.5 \%$ Dabco $(1,4$ diazobicyclo (2.2.2] octane; Sigma, St Louis, $\mathrm{MO}$ ) in glycerol: PBS $(9: 1 \mathrm{v} / \mathrm{v})$, final $\mathrm{pH}$ of 8.6 . The presence or absence of an acrosome reaction was evaluated by counting at least 100 spermatozoa under a fluorescence microscope at $\times 400$ magnification. Spermatozoa were incubated with equal volumes of $0.25 \%$ HSA in physiological saline as a negative control. Heatinactivated human follicular fluid, final dilution 1:4, was also used in some experiments.

\section{Spermatozoa penetration assay}

The effect of PAF on penetration of zona-free hamster oocytes by spermatozoa was assessed using a modification of the methods described by Yanagimachi et al. (1976). Oocytes were retrieved from superovulated, sexually mature Golden Syrian hamsters and treated with $0.1 \%$ hyaluronidase (Sigma) to remove the cumulus and $0.1 \%$ trypsin (Sigma) to remove the zona pellucida. Spermatozoa were capacitated overnight in BWW containing $0.6 \% \mathrm{HSA}$, washed, then resuspended in $10^{-14} \mathrm{~mol} \mathrm{PAF}^{-1}$ in $0.25 \% \mathrm{HSA}$ or in $0.25 \% \mathrm{HSA}$ alone for $30 \mathrm{~min}$. At the end of the preincubation period, spermatozoa were washed, and 5-10 $\times 10^{6}$ motile spermatozoa were added to 25 oocytes in BWW with $0.6 \%$ HSA under oil and incubated for $3 \mathrm{~h}$. At the end of the incubation period, the eggs were removed, mounted on slides and examined at $\times 400$ with phase-contrast optics. The number of oocytes penetrated by at least one spermatozoa was recorded, as was the number of decondensed spermatozoa heads per oocyte.

\section{Electron microscopy}

PAF-treated, acrosome-reacted spermatozoa were fixed for electron microscopy in $2.5 \%$ glutaraldehyde in $0.1 \mathrm{~mol}$ sodium cacodylate $\mathrm{I}^{-1}$ for $\mathrm{1}-2 \mathrm{~h}$ at room temperature, stored overnight at $4^{\circ} \mathrm{C}$, then embedded and prepared for transmission electron microscopy (TEM). Two-hundred spermatozoa were examined by TEM. The patterns described by Yudin et al. (1988) were used to determine which spermatozoa had acrosome reacted. The acrosomal status of the samples of spermatozoa was assessed using FITC-labelled PSA lectin before fixation, and the percentage of acrosome-reacted spermatozoa recorded by TEM correlated with the PSA assessment.

\section{Determination of calcium effects}

The role of calcium in PAF-mediated activation of spermatozoa function was assessed in capacitated, swim-up spermatozoa washed in $\mathrm{BWW}$ containing $\mathrm{Ca}^{2+}$ or in $\mathrm{BWW}$ that was nominally free of $\mathrm{Ca}^{2+}$ (Morales et al., 1989), then incubated with PAF $\left(10^{-14} \mathrm{~mol} \mathrm{I}^{-1}\right)$ for $30 \mathrm{~min}$. Subsequently, spermatozoa were coincubated with hamster oocytes in BWW with or without $\mathrm{Ca}^{2+}$ for $3 \mathrm{~h}$. The SPA was scored and the acrosomal status determined using spermatozoa that were left after removal of eggs for SPA.

\section{Statistical analysis}

One-way and repeated measures analysis of variance, and paired comparisons were used to evaluate concentration and time effects on the acrosome reaction. Student's $t$ tests with Bonferroni adjustment of the $P$ value were used to compare 


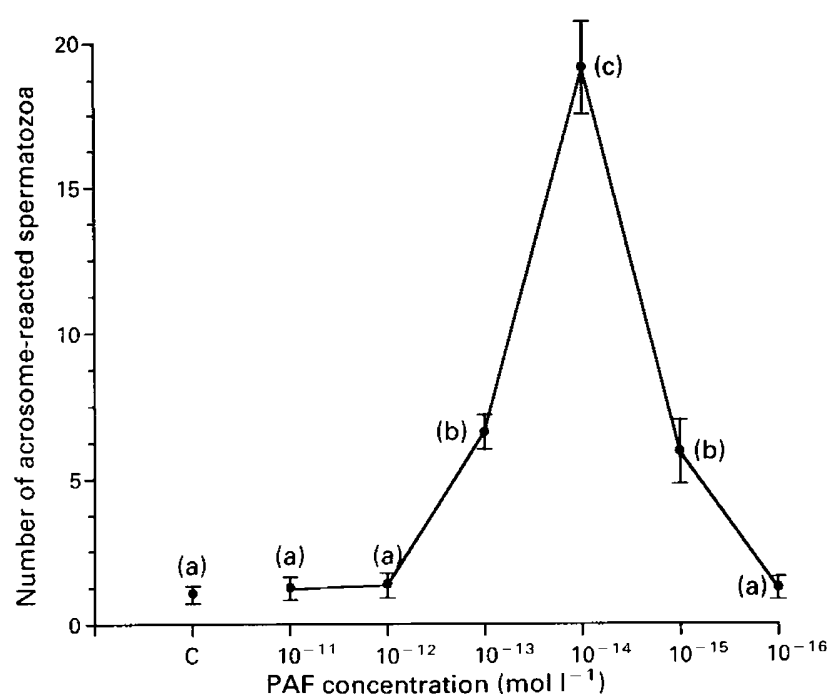

Fig. 1. The effect of decreasing concentrations of platelet-activating factor (PAF) on the acrosomal status of human spermatozoa. PAF was resuspended in $0.25 \%$ human serum albumin (HSA) to give a final concentration of $10^{-11}$ to $10^{-16} \mathrm{~mol} \mathrm{l^{-1 }}$. Each point represents the mean \pm SEM for the number of acrosome-reacted spermatozoa per 100 cells counted from each of nine donors. Values with different letters are significantly different from each other $(P<0.05)$; values with the same letters were not significantly different.

treatment and control groups when one-way analysis of variance was done. An unpaired Student's $t$ test was used to examine statistically the effect of PAF, lyso-PAF, CV3988 and follicular fluid on the acrosome reaction of spermatozoa penetration.

\section{Results}

\section{Dose effect of PAF on acrosome reaction}

Addition of PAF to human spermatozoa initiated a concentration-dependent change in the number of spermatozoa that acrosome-reacted during a $30 \mathrm{~min}$ incubation (Fig. 1). Addition of $10^{-11} \mathrm{~mol} \mathrm{PAF} \mathrm{l}^{-1}$ did not increase the number of acrosome-reacted spermatozoa compared with that seen in spermatozoa treated with HSA alone. However, as the concentration of PAF decreased, the number of acrosome-reacted spermatozoa per 100 cells increased, reaching a peak value at $10^{-14} \mathrm{~mol} \mathrm{PAF} \mathrm{l}^{-1}$, a dose similar to physiological concentrations of PAF. The number of acrosome-reacted spermatozoa then decreased as the PAF concentration further decreased, again approaching control values by $10^{-16}$ mol PAF $\mathrm{I}^{-1}$.

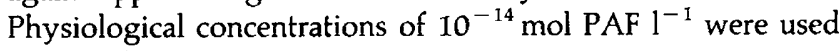
for all subsequent experiments.

\section{Time course of PAF action}

In an effort to determine the time required for PAF to initiate the acrosome response, $10^{-14} \mathrm{~mol} \mathrm{PAF} \mathrm{^{-1 }}$ was added to washed spermatozoa that had been capacitated overnight in BWW with $0.6 \% \mathrm{HSA}$. An aliquot of spermatozoa was removed

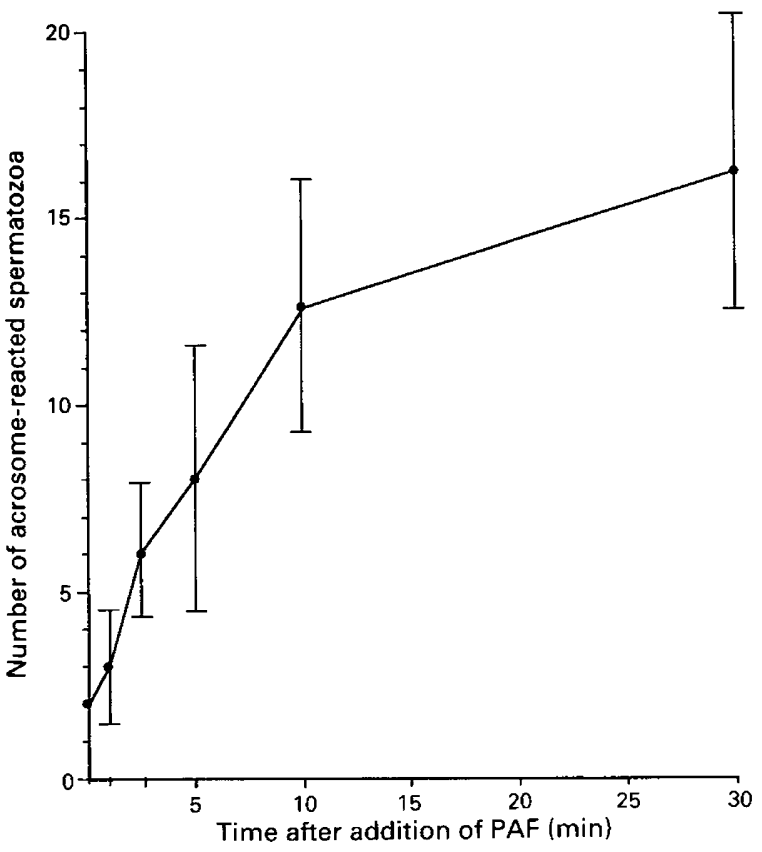

Fig. 2. Time course for PAF induction of the acrosome response. PAF was resuspended in $0.25 \%$ HSA to give a final concentration of $10^{-14} \mathrm{~mol} \mathrm{l}^{-1}$ and incubated with spermatozoa from six donors. At various times an aliquot of the spermatozoa suspension was removed and the acrosomal status of 100 spermatozoa determined. Data points indicate mean $\pm \mathrm{SEM}$.

at $1,2.5,5,10$ and $30 \mathrm{~min}$ and the acrosomal status of the spermatozoa assessed. Repeated measures analysis of variance suggested a significant effect of time on the acrosome reaction $(P=0.0028)$. The number of acrosome-reacted spermatozoa increased three-fold within $2 \mathrm{~min}$ and fourfold within $5 \mathrm{~min}$ of addition of PAF (Fig. 2). The response continued to increase throughout the remainder of the $30 \mathrm{~min}$ incubation. Thirty minute incubations with PAF were therefore performed during all subsequent experiments.

\section{Effect of PAF on noncapacitated spermatozoa}

The previous studies were conducted using spermatozoa that had been capacitated overnight in BWW and 0.6\% HSA. An aliquot of washed, swim-up spermatozoa from ten donors was exposed to PAF or $0.25 \% \mathrm{HSA}$ for $30 \mathrm{~min}$ to determine whether the capacitation step was required for development of the PAF response. The remaining washed spermatozoa were tested with PAF on the following morning. Addition of $10^{-14} \mathrm{~mol} \mathrm{PAF}^{-1}$ did not appear to enhance the number of reacted spermatozoa compared with controls when PAF was added to spermatozoa within $I \mathrm{~h}$ of swim-up ( $1.2 \pm 0.3$ acrosome-reacted spermatozoa, mean $\pm \mathrm{SEM}$, treated with HSA compared with $1.3 \pm 0.5$ acrosome-reacted spermatozoa, mean \pm SEM, treated with PAF within $1 \mathrm{~h}$ after swim-up; data not shown). Failure to induce an acrosome reaction in noncapacitated spermatozoa was also observed following the addition of human follicular fluid (1:4 final dilution) or progesterone (50 $\mathrm{ng} \mathrm{ml}^{-1}$ final concentration). 


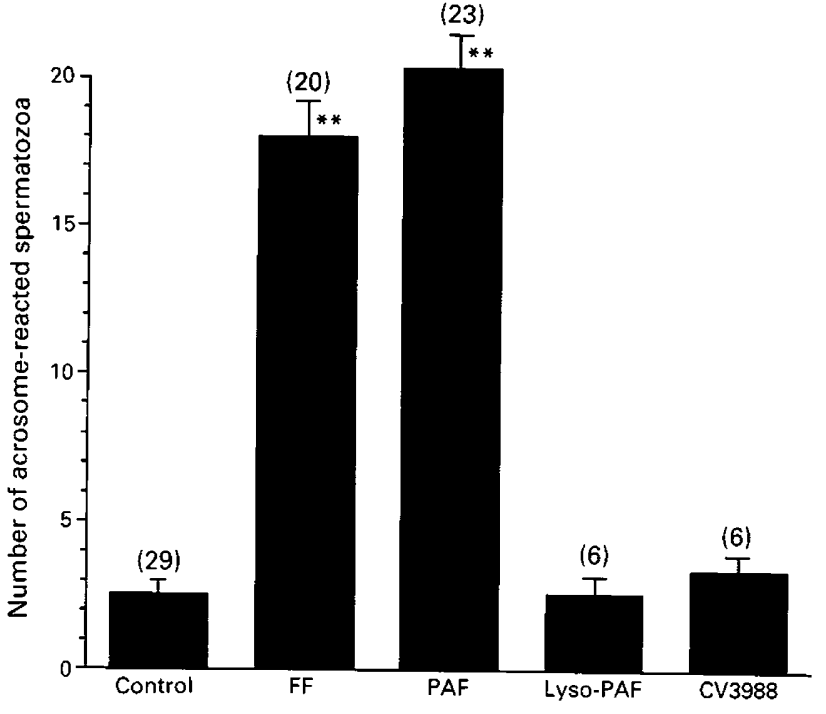

Fig. 3. The effect of human follicular fluid (FF; 1:4 final dilution), PAF $\left(10^{-14} \mathrm{~mol} \mathrm{l}^{-1}\right)$, lyso-PAF $\left(10^{-14} \mathrm{~mol} \mathrm{l}^{-1}\right)$ and CV3988 $\left(10^{-12} \mathrm{~mol} \mathrm{I}^{-1}\right)$ on the acrosomal status of capacitated spermatozoa. Spermatozoa were incubated with FF, PAF, or lyso-PAF for $30 \mathrm{~min}$ before assessment of the acrosome response or preincubated with CV3988 for 30 min before addition of PAF. The acrosomal status of 100 spermatozoa was assessed in the number of specimens indicated in parentheses above each bar. Bars represent means \pm SEM. Significant differences $(P<0.01)$ between experimental and control values ( $t$ test for unpaired samples) are indicated by asterisks.

\section{Effect of follicular fluid, lyso-PAF and CV3988 on capacitated spermatozoa}

After overnight capacitation, addition of human follicular fluid or $10^{-14} \mathrm{~mol} \mathrm{PAF} 1^{-1}$ for $30 \mathrm{~min}$ significantly increased $(P<0.0001$, Student's $t$ test $)$ the number of acrosome-reacted spermatozoa by eight times compared with HSA-treated controls (Fig. 3). The number of spermatozoa that acrosome-reacted following addition of PAF did not appear to be significantly different from the number of spermatozoa that acrosomereacted following the addition of human follicular fluid, a known inducer of the acrosome reaction. Addition of lyso-PAF $\left(10^{-14} \mathrm{~mol} \mathrm{l}^{-1}\right)$, the PAF synthetic precursor and the PAF degradation product, did not appear to induce an acrosome response. In addition, preincubation of spermatozoa with $10^{-12} \mathrm{~mol} \mathrm{CV} 3988 \mathrm{l}^{-1}$, an inhibitor of PAF, appeared to block initiation of the acrosome reaction following addition of $10^{-14} \mathrm{~mol} \mathrm{PAF}^{-1}$.

\section{Effects of PAF on hamster oocyte penetration}

Addition of PAF ( $10^{-14} \mathrm{~mol} \mathrm{l}^{-1}$ for $\left.30 \mathrm{~min}\right)$ to 25 human spermatozoa significantly enhanced the number of zona-free hamster oocytes penetrated by PAF-treated spermatozoa compared with controls ( $P<0.0001$, Student's $t$ test) (Fig. 4a). Decondensed spermatozoa were observed in $93 \%$ of the hamster oocytes after pretreatment of the spermatozoa with PAF compared with $39 \%$ of oocytes with decondensed spermatozoa following incubation in HSA. Pretreatment of human

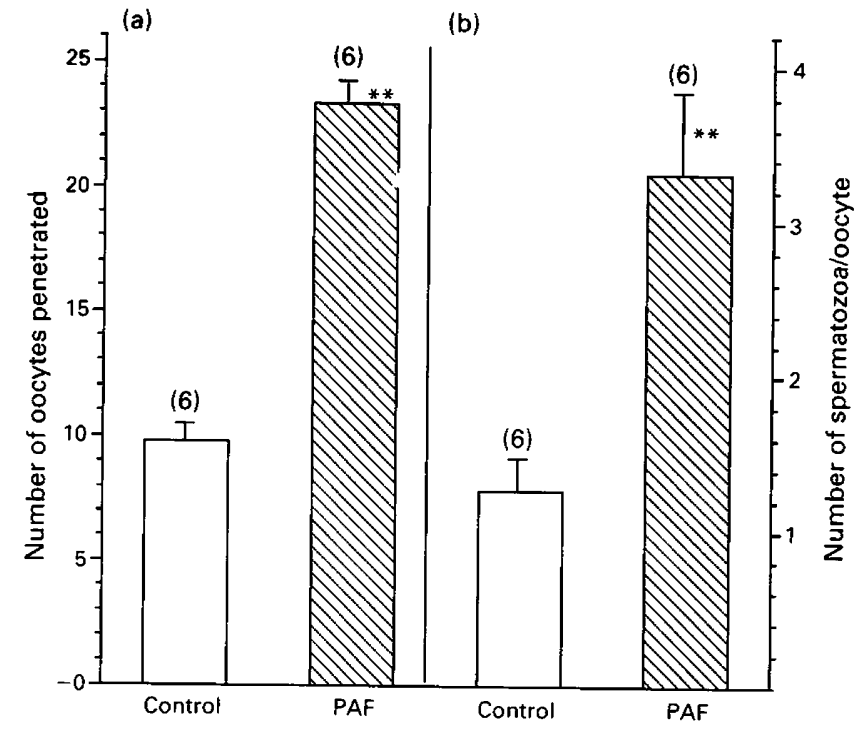

Fig. 4. (a) The effect of PAF $\left(10^{-14} \mathrm{~mol}^{-1}\right)$ on the number of zona-free hamster oocytes penetrated by human spermatozoa $\left({ }^{* *} P<0.0001\right.$; unpaired $t$ test) and (b) on the number of decondensed sperm heads observed per oocyte $\left({ }^{* *} P=0.003\right.$; unpaired $t$ test) during the sperm penetration assay (SPA). Twenty-five hamster oocytes were incubated with an aliquot of PAF-treated or untreated spermatozoa. Bars represent means \pm SEM for six samples.

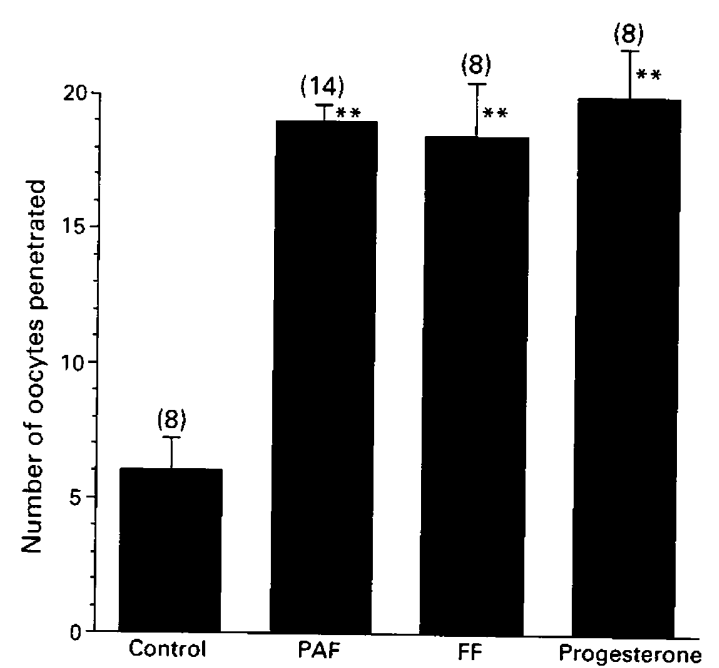

Fig. 5. The effect of PAF $\left(10^{-14} \mathrm{~mol} \mathrm{l}^{-1}\right)$, human follicular fluid (FF 1:4 final dilution) and progesterone $\left(50 \mathrm{ng} \mathrm{ml}^{-1}\right)$ on the number of human spermatozoa penetrating hamster oocytes. Twenty-five oocytes were assessed with treated or untreated spermatozoa from the number of donors shown in parentheses above each bar. Bars represent means \pm SEM; asterisks indicate significant differences between experimental and control groups $(P<0.001)$.

spermatozoa with PAF also increased the number of decondensed spermatozoa heads per egg 2.5 -fold (Fig. $4 \mathrm{~b}$ ) compared with HSA controls $(P<0.003$ Student's $t$ test).

In a subsequent study, the effect of PAF $\left(10^{-14} \mathrm{~mol}^{-1}\right.$ for $30 \mathrm{~min}$ ) on oocyte penetration was compared with the effects of human follicular fluid (FF; 1:4 final dilution) and $50 \mathrm{ng}$ progesterone $\mathrm{ml}^{-1}$ (Fig. 5). Treatment of human spermatozoa 
Table 1. The effect of calcium on the percentage of oocytes containing decondensed sperm heads during the spermatozoa penetration assay (SPA) and on the percentage of acrosome-reacted (AR) spermatozoa after PAF treatment ${ }^{\mathrm{a}}$

\begin{tabular}{|c|c|c|c|c|c|c|}
\hline \multirow[b]{2}{*}{ Event } & \multicolumn{3}{|c|}{$+\mathrm{Ca}^{2+}$} & \multicolumn{3}{|c|}{$-\mathrm{Ca}^{2+}$} \\
\hline & Control & $\mathrm{FF}^{\mathrm{b}}$ & $\mathrm{PAF}^{b}$ & Control & FF & PAF \\
\hline AR (\% reacted) & $2.5 \pm 2.0^{c}$ & $21.0 \pm 1.4^{\mathrm{d}}$ & $23.0 \pm 0.9^{\mathrm{d}}$ & $3.0 \pm 0.4$ & $12.5 \pm 1.3^{\text {de }}$ & $2.5 \pm 0.4^{\mathrm{e}}$ \\
\hline SPA (\% penetrated) & $33.3 \pm 2.1$ & $73.8 \pm 2.6^{\mathrm{d}}$ & $69.8 \pm 1.8^{\mathrm{d}}$ & $14.5 \pm 1.8^{\mathrm{e}}$ & $23.2 \pm 1.1^{\mathrm{de}}$ & $21.7 \pm 1.7^{\mathrm{d}}$ \\
\hline
\end{tabular}

aSpermatozoa from the same donor were analysed in triplicate on three separate occasions.

'FF: follicular fluid; PAF: platelet-activating factor.

'Results are presented as means \pm SEM.

${ }^{d}$ Experimental value was significantly different from control value $(P<0.01$; Student's $t$ test).

'Experimental value in the absence of calcium is significantly different from the experimental value in the presence of calcium $(P<0.01$; Student's $t$ test).

with PAF, follicular fluid and progesterone all appeared to significantly enhance the number of hamster eggs penetrated ( $76 \%, 74 \%$ and $80 \%$, respectively) compared with diluenttreated controls ( $24 \%$ of oocytes penetrated) when analysed using one-way analysis of variance and unpaired $t$ tests with Bonferroni adjustments ( $P<0.001$ for PAF-, follicular fluid-, or progesterone-enhanced penetration compared with penetration of hamster oocytes by diluent-treated control spermatozoa). The effect of follicular fluid on enhancement of oocyte penetration did not appear to be significantly different from the effects of progesterone or PAF $(P>0.05)$.

\section{Role of calcium in PAF effects}

The effect of calcium on follicular fluid- and PAF-induced changes in the acrosome reaction and in the sperm penetration assay was assessed using spermatozoa that had undergone an overnight capacitation in BWW that contained calcium or was nominally calcium free (Table 1 ). Follicular fluid and PAF significantly increased $(P<0.01$; Student's $t$ test) the acrosome reaction, by 8.2 - and 9.2 -fold, respectively, in the presence of calcium. However, in the relative absence of calcium, follicular fluid increased the percentage of spermatozoa that had lost their acrosomes by four times, whereas PAF failed to induce any increase in the acrosome reaction compared with that seen in control spermatozoa incubated in BWW with or without calcium. The increase in the acrosome reaction induced by follicular fluid in the nominal absence of calcium was significantly less $(P<0.01$; Student's $t$ test) than the acrosome reaction that was induced by follicular fluid in the presence of calcium.

When the effect of calcium on follicular fluid- and PAFinduced changes in the sperm penetration assay was examined (Table 1), the percentage of eggs containing decondensed spermatozoa heads increased 2.2-fold and 2.1-fold, respectively, in the presence of calcium. There was no significant difference between changes induced by follicular fluid in the sperm penetration assay and PAF-induced increases. In the absence of calcium, addition of follicular fluid and PAF also significantly increased $(P<0.01$; Student's $t$ test) the percentage of eggs containing decondensed spermatozoa heads compared with control samples incubated in BWW that did not contain calcium (1.6-fold and 1.5-fold increases after FF and PAF treatment). However, the percentage of eggs containing decondensed spermatozoa heads was significantly lower $(P<0.01)$ in the nominal absence of calcium compared with the percentage in the presence of calcium after addition of both follicular fluid and PAF. Control values in the absence of calcium were also significantly lower $(P<0.01)$ than control values in the presence of calcium.

\section{Evaluation of the PAF-induced acrosome reaction by electron microscopy}

Treatment of spermatozoa with physiological concentrations of PAF induced changes in the acrosomal region of the spermatozoa head that appeared to be morphologically normal (Fig. 6) and were similar to those seen using follicular fluid (data not shown). Twenty-three per cent of the PAF-treated spermatozoa, $19 \%$ of the HFF, and $4 \%$ of the nontreated spermatozoa were acrosome reacted as confirmed with TEM and lectin binding.

\section{Discussion}

Platelet-activating factor is a unique alkyl-ether phosphocholine that appears to be involved in a wide spectrum of physiological events. Not only does it influence various pathophysiological responses associated with the inflammatory cascade as well as a multiplicity of physiological processes, but PAF also appears to play a significant role in most aspects of human reproduction. In the female, PAF modulates reproductive events from ovulation to parturition. Although less is known regarding the role of PAF in male reproduction, PAF is thought to be present in spermatozoa (Kumar et al., 1988; Minhas et al., 1988; Angle et al., 1991) and is involved in certain aspects of sperm function including induction of the acrosome reaction (this study), the ability of spermatozoa to penetrate zona free hamster eggs (this study) and possibly spermatozoa motility (Ricker et al., 1989; Helstrom et al, 1991), although there are contradictory data regarding this final point (Angle et al., 1991).

A number of variables may account for the reported differences in the literature with regard to the role of PAF in mediating sperm function. The method of isolating and quantifying PAF before use may modify its perceived dose-response effects. In addition, there appears to be a great deal of interlaboratory variation regarding the source and concentration of 

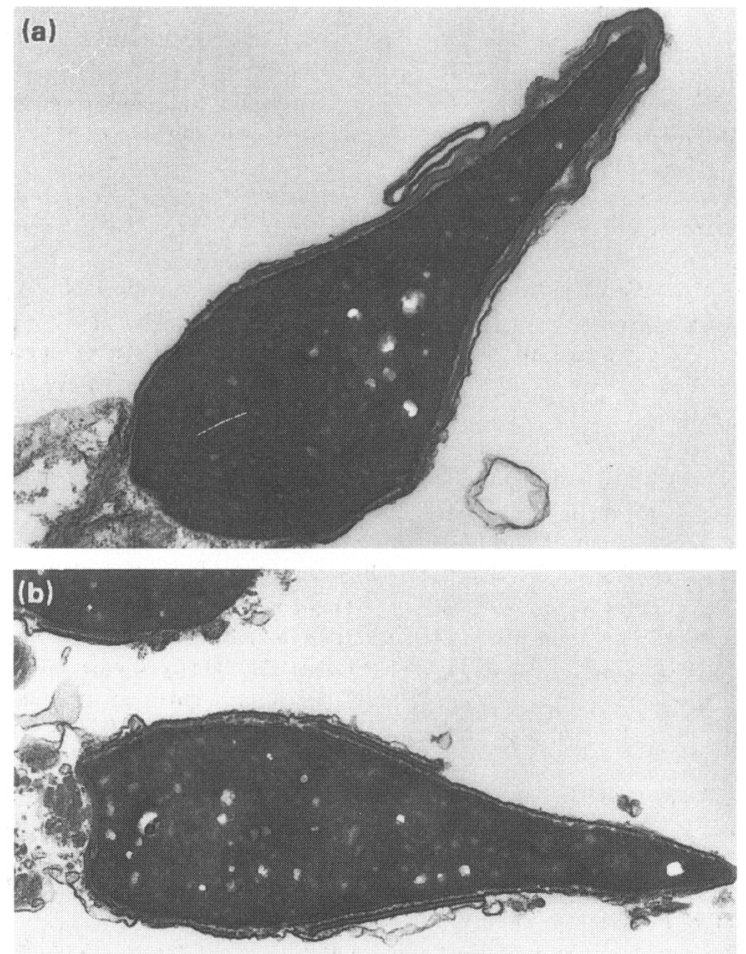

Fig. 6. Morphology of the human spermatozoon acrosome (a) before stimulation and (b) after stimulation by PAF $\left(10^{-14} \mathrm{~mol} \mathrm{l}^{-1}\right)$.

albumin used to resuspend PAF after purification. Finally, simple factors, such as the use of glass tubes or pipettes during the assay, may decrease the actual amount of PAF exposed to cells or tissues during an assay, as a consequence of the avidity of PAF binding to glass, thus affecting the apparent dose of biologically active PAF. Procedural variations between laboratories and the narrow range of PAF effectiveness may therefore account for reported differences in effects of PAF on sperm function.

PAF also appears to play a vital role in fertilization. Admixture of spermatozoa with PAF antagonists prior to artificial insemination of does inhibits fertilization when structural analogues of PAF are used (Harper et al., 1989). Furthermore, addition of PAF enhances the fertilization rate of mouse oocytes in vitro (Kuzan et al., 1990; Minhas et al., 1989) and administration of CV3988, one of the structural analogues of PAF (Minhas et al., 1989) or WEB 2086 (Kuzan et al., 1990), significantly decreases fertilization rates in vitro. As shown in this study, preincubation of human spermatozoa with PAF during the spermatozoa penetration assay increased both the number of decondensed sperm heads found per egg and the number of eggs containing decondensed spermatozoa. As spermatozoa from subfertile men contain significantly different amounts of PAF from those of normal donors (Angle et al., 1991), these data support a role for PAF in normal sperm function and implicate a direct role for PAF in the fertilization process.
PAF that participates in the fertilization process may arise from one of several sources. PAF may come from the spermatozoa, since at least three groups have demonstrated that spermatozoa contain measurable amounts of PAF (Kumar et al., 1988; Minhas et al., 1988; Angle et al., 1991). Thus, PAF present in spermatozoa may play a primary role in intracellular biochemistry of spermatozoa or may be released to act as a paracrine or autocrine mediator. Alternatively, PAF may arise from some component of the maternal system such as the uterus or ovary. Thus, although several studies have suggested that PAF is rarely found in either mouse or human unfertilized oocytes (Angle et al., 1988b; Collier et al., 1990; Punjabi et al., 1990), PAF may be produced by the cumulus cells surrounding the newly ovulated oocyte in concentrations high enough to induce a physiological response in the spermatozoa as they transverse towards the zona pellucida. PAF has also been detected in uterine tissue of rats (Yasuda et al., 1986), rabbits (Yasuda et al., 1986; Angle et al., 1988a) and humans (Alecozay et al., 1989) and specifically in stromal but not glandular cells of the human uterus (Alecozay et al., 1989). Consequently several different reproductive tissues and cell types apparently synthesize PAF.

However, even though PAF has been detected in several reproductive tissues, the kinetics of intracellular PAF synthesis, release and degradation remains relatively unknown. In some cell types, much of the intracellular PAF appears to remain within the cell rather than being released into the extracellular environment (Braquet et al., 1987). Thus, although PAF has been found in spermatozoa, follicular fluid, the ovary and the uterus, whether the PAF from any of these sources may affect other cells of the reproductive tract in vivo remains relatively unexplored. Consequently, the role of PAF in intercellular communication, and thus its regulatory role in the fertilization process, has yet to be defined.

The precise mechanism whereby PAF induces an acrosomal response in spermatozoa is also unknown, but may be mediated via a membrane-associated PAF receptor. PAF receptors have been demonstrated in other cell types: high-affinity receptors have been found in human and rabbit platelets; human, bovine and guinea-pig neutrophils; rabbit and guinea-pig ileum; and human and guinea-pig lung membrane preparations (for references, see reviews by O'Flaherty, 1987 and Braquet et al., 1987). It has been proposed that there are two PAF receptors in the neutrophil, one specific for acyl-PAF derivatives and one for alkyl-PAF derivatives (Levi et al., 1989). Furthermore, monovalent as well as divalent cations and CTP have been shown to enhance the specific binding of PAF to its receptor in the platelet membrane system (Hwang et al., 1986).

The data presented here reveal that the effects of PAF on the acrosome reaction and spermatozoa penetration of hamster oocytes are, at least in part, mediated by calcium, as the PAF-induced acrosome reaction was completely inhibited and spermatozoa penetration significantly depressed in the nominal absence of calcium in this study. Furthermore, while the acrosomal response to follicular fluid was not completely eliminated by removing calcium from the culture media, it was significantly decreased. This result suggests that the response to follicular fluid may also be regulated by calcium. The continued effect of follicular fluid on the acrosome response in the nominal absence of calcium may reflect additional exogenous calcium present in the follicular fluid itself. Thus PAF and human follicular fluid 
may mediate the human acrosome reaction by similar calciumdependent mechanisms.

PAF stimulates other known target cells to take up extracellular calcium, alter their concentration of CAMP in such cells, and turn over phosphatidylinositols (for review see O'Flaherty, 1987). PAF also stimulates GTPase activity in human platelets or their isolated membranes (Avdonin et al., 1985). Many actions of PAF in other cell types require extracellular calcium and are inhibited by calcium antagonists (O'Flaherty, 1987). In addition to its role in the mobilization of extracellular calcium, PAF also induces the release of calcium from intracellular stores (Ingraham et al., 1982). Consequently, it is possible that PAF may interact with specific receptors on the plasma membrane of the spermatozoa to induce a calcium influx, or mobilization of intracellular calcium, although definitive studies demonstrating an increase in intracellular calcium of spermatozoa after exposure to PAF have yet to be done.

In conclusion, these studies suggest that a better understanding of the role of PAF in the physiology of spermatozoa and spermatozoa-oocyte interactions may provide keys to new approaches to male contraception, and to the therapeutic use of PAF during the treatment of clinically subfertile spermatozoa during procedures such as in vitro fertilization.

This work was supported in part by a REAC grant, MSC13, from the School of Medicine, University of California, San Francisco, and presented in part at the 45th Annual Meeting of the American Fertility Society, San Francisco, California, November 1989. We would like to thank B. Munn at the University of California, Davis, Department of Medical Pathology, for embedding and processing the spermatozoa for transmission electron microscopy and for his assistance in microscopic examination. We would also like to thank S. Meizel from the University of California, Davis, for his suggestions and comments.

\section{References}

Abisogun AO, Braquet T and Tsafriri A (1989) The involvement of platelet activating factor in ovulation Science 243 381-384

Acker G, Hecquet F, Etienne A, Braquet P and Mencia-Huerta JM (1988) Role of platelet-activating factor $(\mathrm{Paf})$ in ovoimplantation in the rat: effect of the specific Paf antagonist, BN 52021 Prostaglandins 35 233-241

Acker G, Braquet P and Mencia-Huerta JM (1989) Role of platelet-activating factor (Paf) in the initiation of the decidual reaction in the rat Journal of Reproduction and Fertility $85623-629$

Alecozay AA, Casslén BG, Riehl RM, DeLeon FD, Harper MJK, Silva M, Nouchi T and Hanahan DJ (1989) Platelet activating factor (Paf) in human luteal phase endometrium Biology of Reproduction 41 578-586

Angle MJ, Jones MA, McManus LM, Pinckard RN and Harper MJK (1988a) Platelet-activating factor in the rabbit uterus during early pregnancy joumal of Reproduction and Fertility 83 711-721

Angle MJ, Byrd W and Johnston JM (1988b) Embryonic production of plateletactivating factor in culture. In Proceedings of the Forty-Fourth Annual Meeting of the American Fertility Society, Atlanta pp S96. The American Fertility Society, Birmingham

Angle MJ, Tom R, Khoo D and McClure D (1991) Platelet activating factor (Paf) in spermatozoa from fertile and subfertile men Fertility and Sterility 56 314-318

Avdonin PV, Svitina-Ulitini IV and Kulikov VI (1985) Stimulation of highaffinity hormone-sensitive GTPase of human platelet by 1-O-akyl-2-Oacetyl-sn-glyceryl-3-phosphocholine (platelet activating factor) Biochemical and Biophysical Research Communications 131 307-313

Braquet P, Touqui L, Shen TY and Vargaftig BB (1987) Perspective in platelet activating factor research Pharmacology Reviews 39 97-145
Collier M, O'Neill C, Ammit AJ and Saunders DM (1990) Measurement of human embryo-derived platelet-activating factor ( $P a f$ ) using a quantitative bioassay of platelet aggregation Human Reproduction 5 323-328

Cross NL, Morales P, Overstreet JW and Hanson FW (1986) Two simple methods for detecting acrosome-reacted human spermatozoa Gamete Research 15 213-226

Geissler FT, Kuzan FB, Faustman EM and Henderson WR (1989) Lipid mediator production by post-implantation rat embryos in vitro Prostaglandins 38 145-155

Harper MJK, Woodard DS and Norris CJ (1989) Spermicidal effect of antagonists of platelet-activating factor Fertility and Sterility 51 890-895

Helstrom WJG, Wang R and Sikka SC (1991) Platelet-activating factor stimulates motion parameters of cryopreserved human spermatozoa Fertility and Sterility $56768-770$

Hwang SB, Lam M-H and Pong S-S (1986) Ionic and GTP regulation of binding of platelet-activating factor to receptors and platelet-activating factorinduced activation of GTPase in rabbit platelet membranes journal of Biological Chemistry 261 532-537

Ingraham LM, Coates TD, Allen JM, Higgens CP, Baehner RL and Boxer LA (1982) Metabolic, membrane, and functional responses of human polymorphonuclear leukocytes to platelet-activating factor Blood 59 1259-1266

Johnston JM, Maki N, Angle MJ and Hoffman DR (1990) Regulation of the arachidonic acid cascade and Paf metabolism in reproductive tissues. In Eicosanoids in Reproduction pp 5-37 Ed. MD Mitchell. CRC Press, Inc., Boston

Kumar R, Harper MJK and Hanahan DJ (1988) Occurrence of platelet-activating factor in rabbit spermatozoa Archives of Biochemistry and Biophysics $\mathbf{2 6 0}$ $497-502$

Kuzan FB, Geissler FT and Henderson WR (1990) Role of spermatozoal plateletactivating factor in fertilization Prostaglandins 39 61-74

Levi R, Genovese A and Pinckard RN (1989) Alkyl chain homologs of plateletactivating factor and their effects on the mammalian heart Biochemistry and Biophysics Research Communications 161 1341-1347

Minhas BS, Kumar R, Dodson MG, Palmer TV, Harrill JL and Robertson JL (1988) The presence of platelet activating factor (Paf)-like activity in human spermatozoa and its implications concerning male infertility. In Proceedings of the Forty-Fourth Annual Meeting of the American Fertility Society, Atlanta pp S22. The American Fertility Society, Birmingham

Minhas BS, Kumar R, Ricker DD, Roudebush WE, Dodson MG and Fortunato SJ (1989) Effects of platelet activating factor on mouse oocyte fertilization in vitro American Journal of Obstetrics and Gynecology 161 1714-1717

Morales P, Cross NL, Overstreet JW and Hanson FW (1989) Acrosome-intact and acrosome-reacted human spermatozoa can initiate binding to the zona pellucida Developmental Biology 133 385-392

O'Flaherty JT (1987) Platelet-activating factor: mechanisms of cellular activation. In Platelet-Activating Factor and Related Lipid Mediators, pp 283-298 Ed. F Snyder. Plenum Press, New York

O'Neill C (1985a) Thrombocytopenia is an initial material response to fertilization in mice Journal of Reproduction and Fertility 73 559-566

O'Neill, C (1985b) Examination of the causes of early pregnancy associated thrombocytopenia in mice Joumal of Reproduction and Fertility 73 567-577

O'Neill C (1985c) Partial characterization of the embryo-derived plateletactivating factor in mice Journal of Reproduction and Fertility 75 375-380

O'Neill C (1987) Embryo-derived platelet-activating factor: a pre-implantation embryo mediator of maternal recognition of pregnancy Domestic Animal Endocrinology 4 69-86

O'Neill C and Saunders DM (1985) Assessment of embryo quality Lancet ii 1035-1036

O'Neill C, Gidley-Baird AA, Pike IL, Porter RN, Sinosich MJ and Saunders DM (1985) Maternal blood platelet physiology and luteal-phase endocrinology as a means of monitoring pre- and postimplantation embryo viability following in vitro fertilization Journal of In Vitro Ferfilizaton and Embryo Transfer 2 $87-93$

O'Neill C, Gidley-Baird AA, Pike IL and Saunders DM (1987) A bio-assay for embryo-derived platelet-activating factor as a means of assessing quality and pregnancy potential of human embryos Fertility and Sterility 47 969-975

O'Neill C, Collier M, Ryan JP and Spinks NR (1989) Embryo-derived plateletactivating factor Journal of Reproduction and Fertility Supplement 37 19-27

Orozco C, Perkins T and Clarke FM (1986) Platelet-activating factor induces the expression of early pregnancy factor activity in female mice Journal of Reproduction and Fertility $\mathbf{7 8} 549-555$

Punjabi U, Vereecken A, Delbeke L, Angle MJ, Gielis M, Gerris J, Johnston JM and Bayaert PH (1990) Embryo-derived platelet activating factor, a marker 
of embryo quality and viability following ovarian stimulation for in vitro fertilization Joumal of In Vitro Fertilization and Embryo Transfer 7 321-325

Rabinovici J and Angle MJ (1991) Platelet-activating factor induces progesterone secretion and changes in morphological appearance in luteinizing granulosa cells in vitro Fertility and Sterility 55 1106-1111

Ricker DD, Minhas BS, Kumar R, Robertson JL and Dodson MG (1989) The effects of platelet activating factor on the motility of human spermatozoa Fertility and Sterility $\mathbf{5 2} 655-658$

Rouser G, Siakotos AN and Fleischer S (1966) Quantitative analysis of phospholipids by thin-layer chromatography and phosphorus analysis of spots Lipids 1 85-86
Spinks NR and O'Neill C (1988) Antagonists of embryo-derived plateletactivating factor prevent implantaton of mouse embryos Journal of Reproduction and Fertility 84 89-98.

Yanagimachi R, Yanagimachi H and Rogers BJ (1976) The use of zona-free animal ova as a test system for the assessment of the fertilizing capacity of human spermatozoa Biology of Reproduction 15 471-476

Yasuda K, Satouchi K and Saito K (1986) Platelet-activating factor in normal rat uterus Biochemical and Biophysical Research Communications 138 1231-1236

Yudin AI, Gottlieb W and Meizel S (1988) Ultrastructural studies of the early events of the human sperm actosome reaction as initiated by human follicular fluid Gamete Research 20 I1-24 